\title{
A Epidemiologia nas Políticas, Programas e Serviços de Saúde
}

A Epidemiologia é um dos pilares da Saúde Pública, e como tal deve estar estreitamente incorporada às políticas, programas e serviços públicos de saúde. No Brasil, a criação e o processo de fortalecimento e consolidação do Sistema Único de Saúde (SUS) vem permitindo a garantia da saúde como direito constitucional, e a Saúde Pública vem ampliando consideravelmente o seu espaço dentro desse sistema.

Nos últimos anos, alguns dos principais desafios técnicos impostos ao SUS foi o desenvolvimento de mecanismos para o seu aperfeiçoamento gerencial, a ampliação do escopo de atuação da Vigilância à Saúde, e a capilarização das ações com vistas à promoção e atenção integral à saúde de modo efetivo para todos os segmentos da população, em especial os mais carentes. Para tal, têm se intensificado a descentralização administrativa e financeira na direção dos estados e municípios, bem como a redefinição das atribuições da Vigilância à Saúde, e a incorporação da epidemiologia às práticas rotineiras dos serviços. Nesse processo, cada vez mais intensamente vêm se utilizando e requerendo informações epidemiológicas em nível regional ou local, tanto dos problemas de saúde existentes quanto dos seus determinantes, de modo a se delinear o perfil de necessidades de saúde, fundamental para o atendimento à diretriz de alcance da eqüidade em saúde.

Especificamente no período de 2000 a 2004, que correspondeu ao III Plano Diretor, observou-se um fortalecimento e desenvolvimento expressivo da epidemiologia nos serviços de saúde, em todas as três esferas de governo. A criação da Secretaria de Vigilância em Saúde (SVS) do Ministério da Saúde, MS, que integrou em um único órgão, a vigilância, prevenção e o controle de doenças transmissíveis e nãotransmissíveis, a vigilância ambiental, a sistematização dos dados sobre a saúde produzidos na rede de serviços própria e conveniada ao SUS, e em especial dos sistemas de informação epidemiológica foi um passo significativo dessa consolidação. Desenvolveu-se, também, uma melhor articulação entre a Vigilância Epidemiológica e as políticas de prevenção e controle, a rede de referência de laboratórios de saúde pública, e também as unidades hospitalares. Além disso, implantou-se uma unidade de respostas rápidas para identificação e investigação de surtos, epidemias e eventos inusitados, que vem possibilitando o desencadeamento de medidas oportunas para o controle respectivo. Infelizmente, vem se utilizando apenas parcialmente a capacidade técnico-científica existente no país na formação dos recursos humanos para esta unidade. 
Em resposta à crescente demanda por informações epidemiológicas, podem-se observar avanços importantes no período, como a melhoria da cobertura e qualidade dos sistemas de informação existentes no SUS, e a maior disponibilidade destes sistemas por meio do acesso on-line livre, com níveis de desagregação adequados para as necessidades de gestores, profissionais de saúde, e também da comunidade científica. $\mathrm{O}$ aprimoramento e consolidação das parcerias entre várias instituições produtoras de informação vêm sendo propiciados pela Rede Interagencial de Informações em Saúde (RIPSA), já com 10 anos de existência, e se constituindo em um espaço de troca de experiências e potencialização de produção, padronização e compatibilização das informações, produção e divulgação de indicadores no âmbito do setor saúde.

A intensificação da capacidade de produção e análise de informações epidemiológicas, nos três níveis do sistema de saúde, tem sido evidenciada no grande número de trabalhos apresentados nos Congressos de Epidemiologia e Saúde Coletiva da ABRASCO, bem como de artigos publicados em revistas científicas oriundos dos serviços de saúde. Nesta direção, um marco no incentivo desta produção foi a criação da EXPOEPI, fórum anual específico para divulgação, discussão e premiação de trabalhos e experiências exitosas no campo da epidemiologia nos serviços de saúde. Esta iniciativa da SVS vem estimulando profissionais de estados e municípios na busca de apropriação do conhecimento epidemiológico no desenvolvimento de ações rotineiras sob firmes bases científicas e criatividade técnica.

Apesar dessas constatações, ainda se pode identificar algumas lacunas que precisam ser superadas nos próximos anos. Por exemplo, identifica-se que é necessário o aprimoramento da capacidade dos profissionais em problematizar a sua prática e elaborar perguntas de investigação pertinentes para as políticas de saúde regionais ou locais, e também melhorar a capacidade do uso de métodos de análise epidemiológica. Isto é particularmente notável nos sistemas locais de saúde, e poderá permitir a identificação dos reais problemas de saúde e a definição de sua prioridade política, oferecendo as bases científicas para o planejamento de ações programáticas e o destino dos recursos. É também importante considerar a necessidade de melhoria da qualidade dos registros, especialmente para algumas informações, como as relacionadas a mortes maternas, por acidentes de trabalho e doencas profissionais.Ademais, constata-se que ainda é expressiva a má qualidade do registro de óbitos em municípios pequenos ou mais pobres, o que dificulta, sobremodo, a definição de prioridades nas políticas e na alocação de recursos. Muitas bases de dados ainda se ressentem da falta de número identificador comum que permita a sua junção. Registra-se, ainda, a ausência de diagnóstico de saúde, como nos dados ambulatoriais, que indicam apenas o procedimento, 
limitando o uso desses dados para a produção de indicadores de morbidade úteis para o conhecimento epidemiológico e a definição de políticas adequadas.

Identifica-se, também, a insuficiência de estudos epidemiológicos de base populacional destinados a traçar diagnósticos nacionais da situação de saúde da população brasileira. Nos últimos anos, inquéritos nacionais vêm sendo conduzidos por iniciativa do MS, em parcerias com outras instituições acadêmicas e de serviços, a exemplo do Inquérito Domiciliar sobre Comportamentos de Risco e Morbidade referida de Doenças e Agravos Não-Transmissíveis, Estudo de Soroprevalência de Infecção Chagásica e Hepatites Virais, dentre outros. Estas experiências deverão ser ampliadas para outras áreas relacionadas a problemas de saúde pública relevantes para o país, como o da violência doméstica e urbana, ou para áreas contempladas por programas especiais como a Rede Nacional de Saúde do Trabalhador, que está sendo implantada em todo o território nacional, ou o Programa de Humanização do SUS.

$\mathrm{O}$ aumento da demanda por conhecimento epidemiológico propiciou um rápido desenvolvimento da área de formação de recursos humanos - nas áreas de epidemiologia e vigilância, nos últimos cinco anos. Após intensas discussões e debates criaram-se os mestrados profissionalizantes, e expandiram-se o número e a diversidade temática dos cursos de especialização. Isto tem ocorrido com o incentivo e apoio financeiro e técnico do MS, secretarias de saúde estaduais e municipais. No momento, a SVS está implementando uma rede de formação de recursos humanos em Vigilância em Saúde, que vem se constituindo em importante espaço de articulação e cooperação entre instituições formadoras de diferentes vocações acadêmicas e as regiões do país, o que poderá contribuir para reduzir desigualdades regionais. Parcerias com instituições estrangeiras também vêm sendo realizadas para a formação de pessoal qualificado, o que tem sido objeto de controvérsia em razão da reconhecida qualidade dos programas de formação e de professores em epidemiologia existentes no país.

Foi consenso que a manutenção e o fortalecimento da articulação entre academia e serviços continuam sendo a pedra angular para a superação de muitas das lacunas e insuficiências apresentadas, objeto deste Plano Diretor. Quatro eixos foram então definidos para melhor organizar os problemas, desafios e as proposições definidas: (i) sistemas de informação; (ii) práticas epidemiológicas, subdivididas em análise de situação de saúde, vigilância em saúde e avaliação de programas, serviços e intervenções em saúde; (iii) recursos humanos e (iv) inserção da epidemiologia nas políticas intra e intersetoriais. 


\section{Sistemas de informação}

\subsection{Problemas identificados}

1.1.1 Existência de múltiplos sistemas de informação fragmentados e não integrados, respondendo a diferentes lógicas de formulação e necessidades;

1.1.2 Sobrecarga das esferas municipais e estaduais para a coleta, processamento e cumprimento dos fluxos estabelecidos, em detrimento do aprimoramento da cobertura, qualidade e utilização das informações nos serviços;

1.1.3 Insuficiência da infra-estrutura dos sistemas de informação nas secretarias estaduais e municipais de saúde;

1.1.4 Pobreza da qualidade dos dados e das informações produzidas pelas grandes bases de dados dos sistemas;

1.1.5 Pequeno desenvolvimento de procedimentos de vinculação entre os sistemas;

1.1.6 Insuficiente supervisão e monitoramento continuado dos sistemas de informação;

1.1.7 Pouca flexibilidade dos sistemas de informação em possibilitar às esferas estaduais e municipais a desagregação das informações e a incorporação de variáveis relevantes para a realidade local;

1.1.8 Dificuldade de acesso a dados de saúde e de produção de serviços de saúde dos usuários cobertos por planos de saúde;

1.1.9 Limitação dos sistemas de informação para o conhecimento da situação epidemiológica de subgrupos populacionais específicos como os negros, índios, assentados e quilombolas, seja pela inexistência das variáveis identificadoras ou devido à baixa qualidade do preenchimento dos documentos base de cada sistema;

1.1.10 Insuficiente participação da comunidade científica na definição e operacionalização da política de realização de inquéritos populacionais para monitoramento das Doenças e Agravos não transmissíveis (DANT) e seus 
fatores de risco, doenças transmissíveis e outros agravos;

1.1.11 Limitação dos sistemas de informação disponíveis e não disponíveis nas unidades onde se coletam os dados, tais como unidades de saúde e hospitais;

1.1.12 Deficiências no gerenciamento e atualização de sistemas de informação como o SINAN e SIS-PRENATAL para agravos ou condições de saúde que requerem entradas repetidas de dados nos sistemas (tuberculose, hanseníase, pré-natal);

1.1.13 Pouca utilização do potencial dos bancos de dados nacionais devido a limitações quantitativas e de formação dos recursos humanos;

1.1.14 Dificuldades de acesso e compatibilidade de bases de dados de outros setores e instituições (IBGE, INCRA, IBAMA, Previdência Social, Ministério do Trabalho);

1.1.15 Dificuldades para divulgar as informações produzidas com os sistemas de informação e torná-las acessíveis aos usuários das instâncias de controle social e aos representantes dos movimentos sociais.

\subsection{Ações propostas}

1.2.1 Racionalizar a enorme quantidade de sistemas de informação existentes no Ministério da Saúde/DATASUS, e aprimorá-los de forma a garantir a compatibilidade, integração e vinculação das diversas bases de dados;

1.2.2 Simplificação dos sistemas de informação e inclusão de registro de especificação de Estados e municípios, permitindo, assim, a desagregação das informações, além de flexibilizar a incorporação de registro de dados necessários para a realidade local;

1.2.3 Estabelecimento de filtros estaduais e federais nos sistemas de informação, tendo em vista que não há necessidade de que todos os dados sejam enviados para todos os níveis do sistema de saúde;

1.2.4 Fortalecimento das atividades de supervisão e monitoramento dos sistemas de informações; 
1.2.5 Desenvolvimento de estudos de confiabilidade e validade dos dados dos sistemas de informação;

1.2.6 Realizar oficina para integração e compatibilização entre os sistemas de informação, a ser organizada pelas Comissões de Epidemiologia e Informação em Saúde da ABRASCO, e o Ministério da Saúde;

1.2.7 Articular com a Agência Nacional de Saúde Suplementar (ANS) a regulação e fornecimento das informações epidemiológicas e de produção de serviços dos planos de saúde;

1.2.8 Promover uma discussão nacional sobre inquéritos nacionais para pactuar temas, metodologias, regularidade, abrangência, padronização da organização e gestão de bancos de dados e estratégias de análise; ;

1.2.9 Apoiar o fortalecimento da iniciativa da SVS de constituição da Rede de Formação em Vigilância em Saúde;

1.2.10 Apoiar o estabelecimento de uma política que busque fortalecer e atualizar a infra-estrutura (equipamentos de informática e sistemas) utilizada na área da Epidemiologia e Vigilância em Saúde nas secretarias municipais e estaduais de saúde e no âmbito do Ministério da Saúde;

1.2.11 Priorizar e incentivar a utilização de softwares livres;

1.2.12 Realizar ajustes na gestão dos sistemas de informações, no nível central e local do SUS, com a finalidade de permitir a coleta de dados para o seguimento de doenças e agravos em saúde que requerem registros repetidos de dados nos sistemas;

1.2.13 Criar mecanismos e grupos de trabalho intersetoriais para compatibilizar as bases de dados dos diferentes sistemas de informação do Ministério da Saúde e de outras instituições.

\section{Práticas epidemiológicas - Análise de situação de saúde}

\subsection{Problemas identificados}

2.1.1 Insuficiência da formação dos profissionais de saúde para a utilização de 
métodos de análise apropriados a pequenos números, tais como estudo da situação de saúde em níveis local e distrital, e etnias específicas, entre outras;

2.1.2 Pouca disseminação do uso dos Sistemas de Informação Geográfica, SIG e técnicas de análise de distribuição espacial nos serviços de saúde nas três esferas de governo;

2.1.3 Incipiente desenvolvimento da avaliação de desempenho de programas, ações e serviços de saúde, sendo raros o uso de indicadores de processo, ou a estimativa e divulgação de resultados preliminares que permitam introduzir modificações em etapas oportunas de desenvolvimento das atividades;

2.1.4 Insuficiente avaliação e o monitoramento de desigualdades sociais em saúde;

2.1.5 Dificuldades de cooperação permanente entre universidades e serviços de saúde para o desenvolvimento de pesquisas operacionais;

2.1.6 Pouca valorização e uso insuficiente de estudos descritivos e ou de desenho ecológicos na análise da situação de saúde;

2.1.7 Limitações na divulgação e comunicação dos resultados de estudos e intervenções em saúde para a mídia e a sociedade em geral.

\subsection{Ações propostas}

2.2.1 Apoiar a realização de uma oficina a ser promovida pela Comissão de Epidemiologia da ABRASCO com o objetivo de sistematizar e avaliar os vários métodos utilizados na análise de dados de pequenas populações

2.2.2 Apoiar o desenvolvimento de estratégias de divulgação de resultados de estudos voltadas para as secretarias estaduais e municipais de saúde;

2.2.3 Apoiar a capacitação de profissionais para o desenvolvimento de aplicativos (softwares livres) e o uso de Sistemas de Informação Geográfica, SIG, visando a incorporação e disseminação da análise de distribuição espacial no âmbito dos serviços de saúde;

2.2.4 Promover oficinas para o desenvolvimento de estratégias de construção e análise de indicadores de avaliação de desempenho para o monitoramento 
de intervenções e o seu uso como instrumento de gestão nos diversos níveis do Sistema Único de Saúde;

2.2.5 Implementar estratégias de análise e aplicativos para avaliação de desigualdades em saúde;

2.2.6 Promover estratégias de articulação das redes de formação de Vigilância em Saúde e os pólos de educação permanente e outras instâncias existentes nos Estados para o fortalecimento da cooperação permanente entre serviços e universidades;

2.2.7 Capacitar os gerentes de sistemas e profissionais técnicos de Epidemiologia e Vigilância em Saúde para o uso de dados disponíveis para o monitoramento e a tomada de decisão;

2.2.8 Capacitar profissionais de saúde para a comunicação social da informação epidemiológica.

\section{Vigilância em Saúde}

\subsection{Problemas identificados}

3.1.1 Insuficiente implementação da vigilância ampliada em saúde que inclua as doenças transmissíveis emergentes, as doenças e agravos não transmissíveis (DANT) e a ações da vigilância em saúde ambiental, englobando assim os desastres naturais e ambientais, e as exposições a produtos perigosos;

3.1.2 O Sistema de Vigilância Epidemiológica (SVE) do país não está ainda suficientemente preparado para dar respostas rápidas para situações inusitadas;

3.1.3 Pouca atenção da Vigilância em Saúde a populações especiais, como indígenas, quilombolas e assentados;

3.1.4 Pouca estruturação do sistema de vigilância e monitoramento de DANT nas três esferas de governo;

3.1.5 Insuficiente articulação entre a área de assistência individual e a de Saúde Pública em todas as instâncias de gestão do SUS; 
3.1.6. Insuficiente entrosamento entre a Vigilância Sanitária, a Vigilância Epidemiológica e Vigilância Ambiental nas esferas estadual e municipal.

\subsection{Ações propostas}

3.2.1 Fortalecer a habilidade do SNVS em dar respostas rápidas a emergências epidemiológicas e eventos inusitados por meio de maior utilização da capacidade técnico-científica nacional na formação de pessoal qualificado;

3.2.2 Fortalecer as iniciativas de implantação das políticas de vigilância e promoção de saúde para as DANT em todas as esferas de governo;

3.2.3 Incluir na agenda do SUS a atenção para populações especiais como indígenas, quilombolas e assentados, entre outras;

\section{Avaliação de programas, serviços e intervenções em saúde}

\subsection{Problemas identificados}

4.1.1 Pouco desenvolvimento e utilização de resultados de estudos de avaliação de efetividade, de custo-benefício e de impacto de ações e serviços de saúde;

4.1.2 Insuficiente qualificação dos profissionais de saúde para a avaliação em saúde;

4.1.3 Poucos estudos epidemiológicos para a avaliação de impacto das intervenções;

4.1.4 Pouca utilização dos estudos de meta-análise ou de revisões sistemáticas para subsidiar a tomada de decisões sobre a introdução de novas tecnologias e também para a definição de protocolos assistenciais.

\subsection{Ações propostas}

4.2.1 Capacitar profissionais para a avaliação de efetividade, de custo-benefício e de impacto de ações e serviços de saúde, empregando o raciocínio e o método epidemiológico;

4.2.2 Apoiar a sistematização e divulgação de experiências de avaliação de ações e serviços de saúde; 
4.2.3 Estimular e apoiar a realização e divulgação dos estudos de meta-análise ou de revisões sistemáticas para subsidiar a tomada de decisões, especialmente para a introdução de novas tecnologias e definição de protocolos assistenciais.

\section{Recursos Humanos}

\subsection{Problemas identificados}

5.1.1 Inexistência de um plano de carreira para os profissionais de Epidemiologia e Vigilância em Saúde nas três esferas do SUS;

5.1.2 Baixa freqüência de realização de concursos públicos, grande precarização dos contratos de trabalho, baixos salários e descontinuidade das gestões nas três esferas de governo;

5.1.3 Inadequação dos programas de formação de pessoas com alguns usos desconectados das práticas dos profissionais e com métodos de ensino pouco atrativos e efetivos;

5.1.4 Falta de planejamento e programação periódica das iniciativas de formação dos profissionais de saúde, ocasionando uma sobrecarga de capacitações de curta duração e dificultando as atividades técnicas dos mesmos;

5.1.5 Insuficiente utilização de estratégias de formação, tais como ensino à distância, treinamento em serviço, entre outras;

5.1.6. Pouca inserção de cursos de epidemiologia e vigilância em saúde na programação dos pólos de educação permanente do SUS.

\subsection{Ações propostas}

5.2.1 Apoiar a iniciativa do MS de formulação das diretrizes para um Plano de Carreira do SUS e estimular os Estados e municípios a implantar os seus planos de carreira, cargos e salários, incluindo uma carreira específica para os profissionais de Saúde Coletiva;

5.2.2 Reforçar o concurso público como forma de ingresso no serviço público com o aprimoramento dos mecanismos de seleção para o ingresso de profissionais mais qualificados para a vigilância em saúde; 
5.2.3 Superar a precarização das relações de trabalho no âmbito do SUS e dar agilidade à realização de concursos;

5.2.4 Desenvolver abordagens e metodologias adequadas de formação dos profissionais, levando em conta os problemas e as necessidades reais vivenciadas nos seus processos de trabalho e a utilização de estratégias pedagógicas participativas e problematizadoras;

5.2.5 Incentivar os gestores a realizar planejamento e programação periódica da formação dos profissionais;

5.2.7 Racionalizar os cursos de curta duração de forma a transformá-los em módulos de cursos que titulem como os de especialização;

5.2.8 Promover a utilização e avaliação de estratégias de formação inovadoras como o ensino à distância, treinamento em serviço, etc.;

5.2.9 Estimular uma postura mais ativa dos gestores e profissionais da área da Epidemiologia nos pólos de educação permanente no sentido de priorizar os conteúdos de epidemiologia nos conteúdos curriculares.

\section{Inserção da epidemiologia nas políticas intra e intersetoriais}

\subsection{Problemas identificados}

6.1.1 Insuficiente implantação de políticas intersetoriais de intervenção sobre condicionantes e determinantes de doenças e agravos à saúde das populações;

6.1.2 Falta de aprofundamento na discussão do modelo de Vigilância em Saúde em implementação no país e suas interfaces intra e intersetoriais;

6.1.3 Insuficiente articulação intra-setorial com as áreas da atenção à saúde, ambiental, saúde do índio e outros programas de foco específico;

6.1.4 Insuficiente entrosamento com áreas que desenvolvem políticas prioritárias de governo como o Ministério do Desenvolvimento Social, Ministério das Cidades, Ministério do Meio Ambiente e outros. 


\subsection{Ações propostas}

6.2.1 Estímulo às iniciativas de constituição de grupos de trabalho intersetoriais que possibilitem uma atuação articulada e integrada no enfrentamento de problemas com múltiplas determinações e resolução multisetorial como forma e maior efetividade e racionalidade dos custos;

6.2.2 Realizar oficina para aprofundamento do modelo de Vigilância em Saúde em implementação no país e suas interfaces intra e intersetorial;

6.2.3 Estímulo de criação de grupos de trabalho matriciais intra-setoriais entre as áreas: atenção à saúde, vigilância ambiental, saúde do índio e programas para áreas específicas;

6.2.4 Implementação da política de promoção de saúde do Ministério da Saúde calcada na mudança do modelo assistencial e na construção de modos de vida saudáveis. 